\title{
Perioperative and oncological outcomes following minimally invasive versus open pancreaticoduodenectomy for pancreatic duct adenocarcinoma
}

\author{
Rui Sun ${ }^{1,2,3} \cdot$ Jiawen $\mathrm{Yu}^{1,2} \cdot$ Yifan Zhang $^{3} \cdot$ Zhika Liang $^{4} \cdot$ Xianlin Han $^{1}$ (1)
}

Received: 21 November 2019 / Accepted: 13 May 2020 / Published online: 6 July 2020

(c) The Author(s) 2020

\begin{abstract}
Background The outcomes of minimally invasive pancreaticoduodenectomy have not been adequately compared with those of open pancreaticoduodenectomy in patients with pancreatic ductal adenocarcinoma. We performed a meta-analysis to compare the perioperative and oncological outcomes of these two pancreaticoduodenectomy procedures specifically in patients with pancreatic ductal adenocarcinoma.

Methods Before this study was initiated, a specific protocol was designed and has been registered in PROSEPRO (ID: CRD42020149438). Using the Preferred Reporting Items for Systematic Reviews and Meta-analyses (PRISMA) guidelines, PubMed, EMBASE, Web of Science, Cochrane Central Register, and ClinicalTrials.gov databases were systematically searched for studies published between January 1994 and October 2019. Overall survival, disease-free survival, and time to commencing adjuvant chemotherapy were the primary endpoint measurements, whereas perioperative and short-term outcomes were the secondary endpoints.

Results The final analysis included 9 retrospective cohorts comprising 11,242 patients (1377 who underwent minimally invasive pancreaticoduodenectomy and 9865 who underwent open pancreaticoduodenectomy). There were no significant differences in the patients' overall survival, operative time, postoperative complications, 30-day mortality, rate of vein resection, number of harvested lymph nodes, or rate of positive lymph nodes between the two approaches. However, diseasefree survival, time to starting adjuvant chemotherapy, length of hospital stay, and rate of negative margins in patients who underwent minimally invasive pancreaticoduodenectomy showed improvements relative to those in patients who underwent open surgery.

Conclusions Minimally invasive pancreaticoduodenectomy provides similar or even improved perioperative, short-term, and long-term oncological outcomes when compared with open pancreaticoduodenectomy for patients with pancreatic ductal adenocarcinoma.
\end{abstract}

Keywords Pancreatic ductal carcinoma $\cdot$ Pancreaticoduodenectomy $\cdot$ Laparoscopic surgery $\cdot$ Robotic surgical procedures · Minimally invasive surgery $\cdot$ Meta-analysis

Rui Sun and Jiawen Yu contributed equally to this articleand both should be considered first author.

Electronic supplementary material The online version of this article (https://doi.org/10.1007/s00464-020-07641-1) contains supplementary material, which is available to authorized users.

Xianlin Han

hanxianlin@pumch.cn

1 Department of General Surgery, Peking Union Medical College Hospital, No.1 Shuaifuyuan Wangfujing Dongcheng District, Beijing 100730, China

2 Chinese Academy of Medical Sciences and Peking Union Medical College, Beijing, China
Pancreatic ductal adenocarcinoma (PDAC) is an aggressive malignancy that is the 14th most common cancer and the seventh leading cause of cancer-related mortality worldwide

3 Department of Pediatric Surgery, Capital Institute of Pediatric, Beijing, China

4 Department of Agronomy and Horticulture, University of Nebraska-Lincoln, Lincoln, USA 
[1]. Despite the application of various neoadjuvant and adjuvant treatment protocols, pancreaticoduodenectomy (PD) remains the only curative treatment for patients with adenocarcinoma of the pancreatic head. PD is one of the most complex procedures in gastroenterological surgery, and requires extensive visceral organ dissection and complex reconstructive digestive anastomoses; therefore, it is normally performed using an open approach.

The first laparoscopic PD (LPD) was reported by Gagner and Pomp in 1994 [2], while the first robotic PD (RPD) was described by Giulianotti et al. in 2003 [3]. Since then, minimally invasive PD (MIPD), which encompasses LPD and $\mathrm{RPD}$, is increasingly used worldwide; however, it remains a challenging procedure because of the technical limitations of laparoscopy or robot control as well as the steep learning curve when training physicians. Therefore, the feasibility and safety of the minimally invasive approach remain controversial.

Recent studies [4, 5] and meta-analyses [6, 7] showed that the outcomes of MIPD were similar to or more favorable than open PD (OPD) with respect to the incidence of postoperative morbidity, short-term oncologic outcomes, and long-term overall survival rates. Furthermore, MIPD is associated with lower estimated intraoperative blood loss, shorter length of hospital stay (LOS), higher rate of R0 resection, and the harvesting of a greater number of lymph nodes. However, these studies involved patients with a variety of disease histologies; therefore, objective conclusions regarding the oncologic outcomes of patients with this malignancy, especially PDAC, were unclear. Other related meta-analyses [8,9] involved relatively few patients with PDAC. According to our search, no meta-analysis was performed specifically to investigate the perioperative, shortterm, and long-term oncological outcomes of patients who underwent MIPD for PDAC. In this study, we carefully screened and selected studies that specifically investigated patients with PDAC. The aim of our meta-analysis was to meaningfully assess the perioperative, short-term, and longterm oncologic outcomes of these patients, with the primary investigative endpoints being overall survival (OS), diseasefree survival (DFS), and the time to starting postsurgical adjuvant chemotherapy.

\section{Materials and methods}

Before this study was initiated, we designed a specific protocol which has been registered in PROSEPRO. The ID is CRD42020149438 (details of registration is included in Supplementary Materials). Thus, this study was performed in accordance with the recommendations of the Preferred Reporting Items for Systematic Reviews and Meta-analyses (PRISMA) guidelines [10]. This article does not contain any studies with human participants performed by any of the authors; therefore, there was no requirement for IRB approval.

\section{Data sources and search methods}

We systematically searched the PubMed, EMBASE, Web of Science, Cochrane Central Register, and ClinicalTrials.gov databases for studies published in English between 1994 and October 2019. The search Medical Subject Heading (MeSH) terms were "laparoscopic pancreaticoduodenectomy" OR "robotic pancreaticoduodenectomy" AND "pancreatic ductal adenocarcinoma," as well as all associated entry words retrieved using the MeSH index (details of our search strategy are included in Supplementary Materials). There was no language restriction. We also reviewed the introduction and discussion sections of the retrieved manuscripts, relevant review articles, and published meta-analyses to identify additional trials. Two authors (Rui Sun and Jiawen Yu) independently conducted the literature search, screened the abstracts, and selected the relevant trials.

\section{Inclusion criteria}

Studies published between January 1994 and October 2019 were considered eligible if they met the following inclusion criteria: (1) the investigated population comprised patients with PDAC; (2) the interventions compared were LPD or RPD versus OPD; and (3) the study investigated at least one of the following outcomes: operative time, intraoperative blood transfusion, postoperative morbidity and mortality, LOS, rate of vein and R0 resection, number of lymph nodes retrieved, time to starting adjuvant treatment, DFS, and OS.

\section{Exclusion criteria}

The exclusion criteria are as follows: (1) review articles; (2) meeting abstracts; (3) irrelevant studies such as those that investigated only a single surgical technique; (4) insufficient information available in the English abstract; (5) studies with no comparative data; and (6) studies without PDAC data. If papers had overlapping data, those describing the smaller-scale studies were excluded.

\section{Quality assessment}

We adopted the Newcastle-Ottawa Scale (NOS) [11], which is designed specifically for observational investigations, to assess the quality of the selected studies. The NOS focuses on 3 separate sections of a case-control or cohort study, with the number of stars representing the assessment score. The maximum achievable score under the NOS is 9 stars, including 4 for the selection process, 2 for comparability, and 3 for 
exposure and outcome. A score of $\geq 6$ stars is considered indicative of high quality. Two investigators independently assessed the selected studies.

\section{Data extraction}

Two investigators independently extracted the following information: first author, year of publication, study type, mean age, population size, tumor size, and main outcomes; the latter included operative time, intraoperative blood transfusion, postoperative morbidity and mortality, LOS, rate of vein and R0 resection, number of lymph nodes retrieved, time to adjuvant treatment, DFS, and OS. The evaluators resolved any disputes via consensus during the screening processes.

\section{Statistical analysis}

Statistical analyses were conducted using Review Manager 5.3 (Cochrane Collaboration). The relative risk (RR) and mean difference (MD) with the $95 \%$ confidence interval (CI) were used as the measures of dichotomous and continuous variables, respectively. Natural logarithm hazard ratios (HRs) and standard errors were used as summary statistics for DFS and OS data. Using the methods of Wan et al. [12] and Luo et al. [13], medians with ranges as well as the mid-quartile range were converted into means with standard deviations. For studies in which natural logarithm HRs and standard errors or corresponding 95\% CIs were not available, estimates from the published survival curves were calculated using the method suggested by Tierney et al. [14]. $P$-values less than 0.05 indicated statistical significance. Heterogeneity was quantified by the $I^{2}$ statistic; a study with an $I^{2}$ less than $50 \%$ was considered to have no heterogeneity, and the fixed effects model was then applied to pool the results; otherwise, the random effects model was used.

\section{Results}

\section{Search results and characteristics of the included studies}

A flowchart of our analysis protocol is shown in Fig. 1. Our analysis included 9 retrospective cohort studies [15-23] comparing MIPD and OPD in a total of 11,242 patients with PDAC (1377 and 9865 underwent MIPD and OPD, respectively). The characteristics and qualities of these 9 studies are listed in Table 1, while the main results are shown in Table 2. All the analysis results including operative time, intraoperative blood transfusion, LOS, postoperative complications and mortality, and short- and long-term oncological outcomes are shown in Figs. 2, 3, 4, and 5. Among the 9 studies, 8 [15, 17-23] compared LPD and OPD and only 1 [16] compared RPD and OPD. The means of the patients' ages in each study ranged from 65.4 to 79.6 years with a majority in both the MIPD and OPD cohorts being of comparable age; only patients of both groups in Chapman et al.'s study (79.6 versus 79.6 years; $P=0.99$ ) [22] were older than those in other studies. There were no significant differences between the proportions of male and female patients with PDCA.

\section{Perioperative outcomes}

\section{Operative time}

Three studies [15, 21, 23] that encompassed 913 patients investigated operative times (including 228 and 685 patients who underwent MIPD and OPD, respectively). The operative time was longer for the MIPD group in 2 of these studies, and was longer for the OPD group in the third. The pooled estimates of these studies showed that the duration of surgery was not significantly different between the MIPD and OPD groups (MD 50.09; 95\% CI - 25.21 to 125.38; $P=0.11)$. The analysis found statistically significant heterogeneity $\left(I^{2}=97 \%\right)$; thus, a random effects model was adopted (Fig. 2A). No differences in the results and no heterogeneity were found on sensitivity analysis.

\section{Intraoperative blood transfusion}

Three studies encompassing 913 patients (228 who underwent MIPD and 685 who underwent OPD) compared intraoperative blood transfusion rates [15, 21, 23]. The pooled results showed a higher rate of intraoperative blood transfusion in the OPD group; the pooled RR $(0.58 ; 95 \%$ CI 0.44 to $0.77 ; P=0.0002$ ) showed a significant difference in the intraoperative blood transfusion rate between the two groups. Heterogeneity was not significant $\left(I^{2}=0 \%\right)$ (Fig. 2B).

\section{LOS}

Six trials $[15,17,20-23]$ with a total of 10,863 patients (1319 and 9544 who underwent MIPD and OPD, respectively) investigated the LOS. Five studies showed the LOS to be significantly lower in the MIPD group, whereas 1 showed it to be lower in the OPD group. The analysis found significant heterogeneity $\left(I^{2}=98 \%\right)$, and a random effects model was adopted. The pooled mean difference $(\mathrm{MD}=-4.66$; $95 \% \mathrm{CI}-9.13$ to $-0.19 ; P=0.04)$ indicated a significantly shorter LOS in the MIPD group (Fig. 2C). The results and heterogeneity were not significantly different in the sensitivity analysis. 
Fig. 1 Flowchart showing the protocol of the meta-analysis. MIPD minimally invasive pancreaticoduodenectomy, $O P D$ open pancreaticoduodenectomy, $P D A C$ pancreatic duct adenocarcinoma

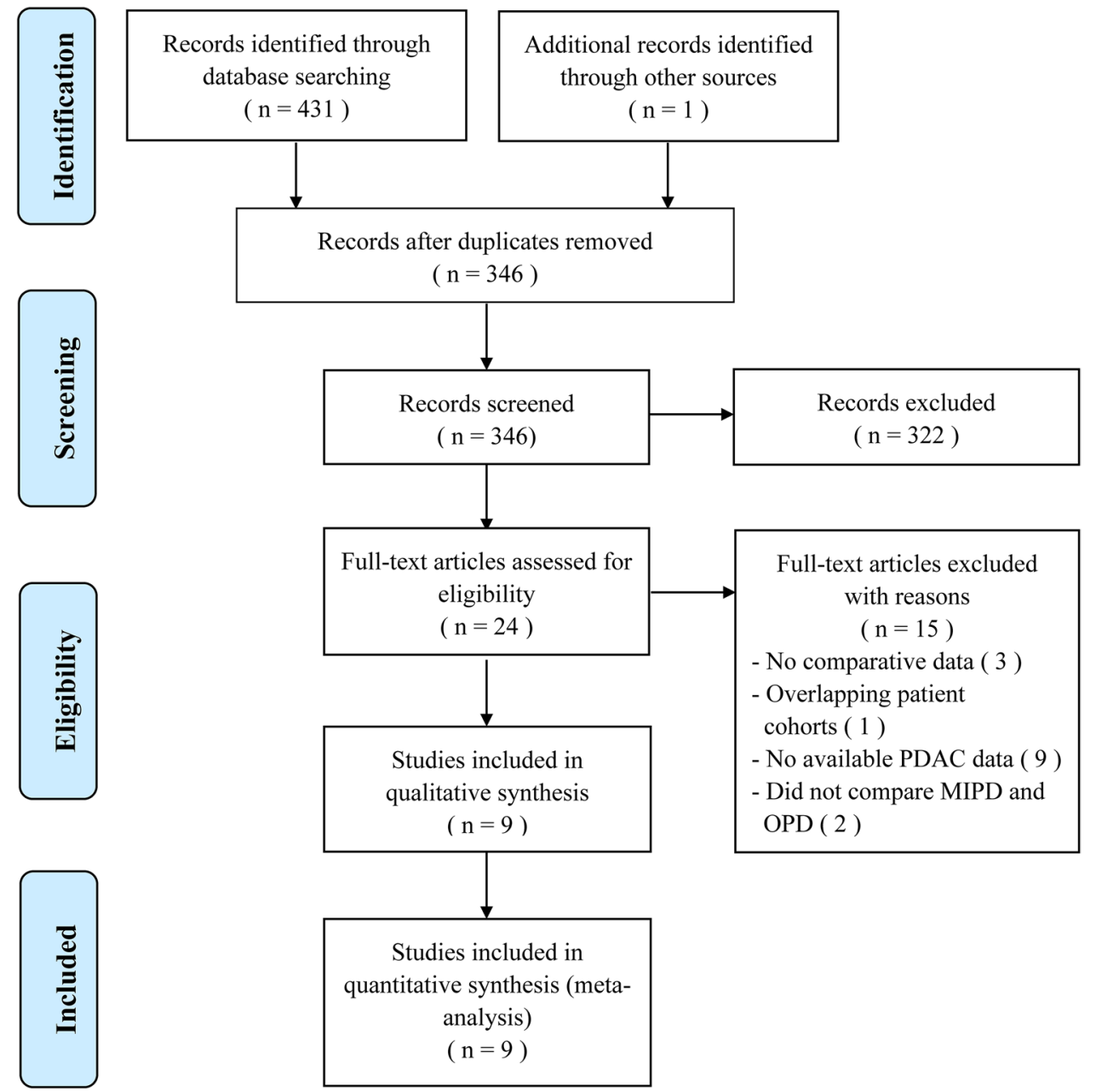

\section{Postoperative complications and 30-day mortality}

Four studies that included 942 patients combined (243 who underwent MIPD and 699 who underwent OPD) [15, 17, 21, 23] examined surgical complications. The pooled surgical complication data revealed no difference between MIPD and OPD (RR $=0.84 ; 95 \%$ CI 0.67 to $1.05 ; P=0.12$, $I^{2}=45 \%$ ) (Fig. 3A). A lack of significant differences was also observed on subgroup analyses of postoperative pancreatic fistulae (3 studies [15, 17, 21], RR $=0.91 ; 95 \% \mathrm{CI}$, 0.56 to $1.46 ; P=0.69, I^{2}=0 \%$ ) (Fig. 3B), delayed gastric emptying (3 studies [15, 17, 21], RR $=0.86 ; 95 \% \mathrm{CI}, 0.33$ to $2.28 ; P=0.77, I^{2}=67 \%$ ) (Fig. $3 \mathrm{C}$ ), and postpancreatectomy hemorrhage (3 studies $[15,17,21], \mathrm{RR}=1.33$; $95 \%$ CI 0.68 to $2.58 ; P=0.4, I^{2}=0 \%$ ) (Fig. 3D). Similarly, no significant difference in postoperative 30-day mortality was detected ( $\mathrm{RR}=1.01 ; 95 \% \mathrm{CI}, 0.73$ to $1.42 ; P=0.93$, $\left.I^{2}=0 \%\right)$ in six studies $[15,17,18,20,22,23]$ that comprised 10,884 patients (1272 and 9612 who underwent MIPD and OPD, respectively) (Fig. 3E).

\section{Short-term oncological outcomes}

\section{Rate of vein resection}

Three studies [15, 21, 23] comprising a total of 913 patients (228 underwent MIPD and 685 underwent OPD) provided data on vein resection; our analysis revealed no difference in the vein resection rate $(\mathrm{RR}=0.96 ; 95 \% \mathrm{CI} 0.76$ to 1.20 ; $\left.P=0.71, I^{2}=0 \%\right)$ (Fig. 4A).

\section{Rate of $\mathrm{R} 0$ resection}

Eight studies [15-18, 20-23] including 11,192 patients (1349 and 9843 underwent MIPD and OPD, respectively) provided data regarding the $\mathrm{R} 0$ resection rate. We found that the R0 resection rate was higher in the MIPD group, with low heterogeneity as shown in a random effects model (RR $=1.06 ; 95 \%$ CI 1.01 to $1.12 ; P=0.02, I^{2}=36 \%$ ) (Fig. 4B). 


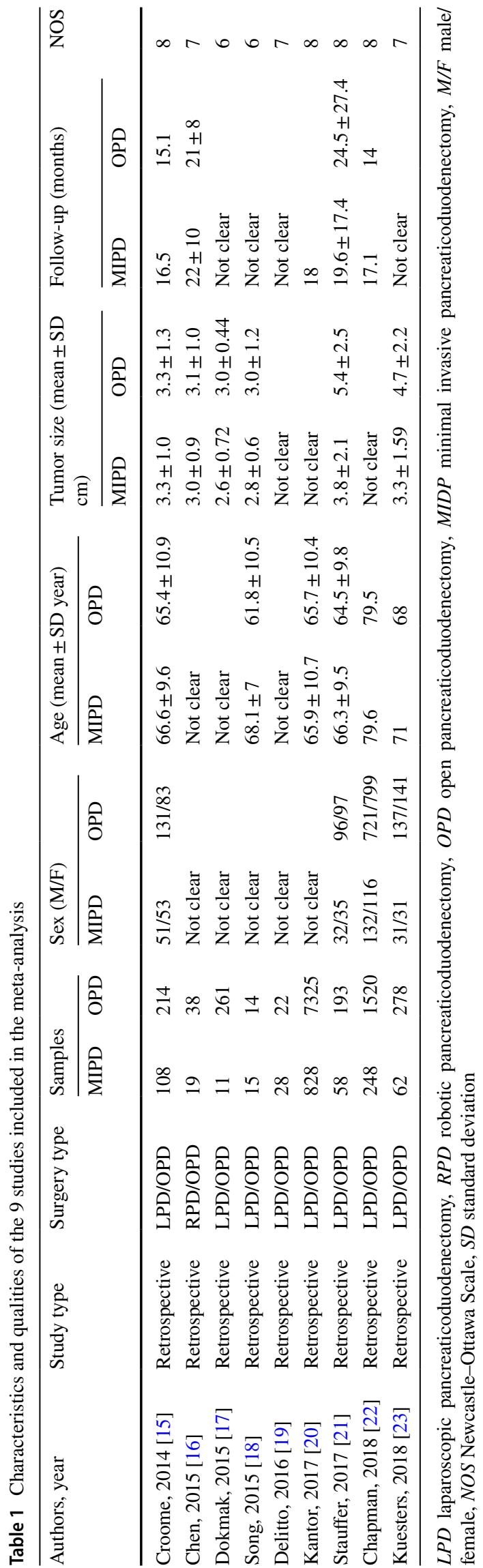

\section{Harvesting and positivity rate of lymph nodes}

The numbers of harvested lymph nodes were measured in 7 studies [15-18, 20, 21, 23] that included 9575 patients (1279 who underwent MIPD and 8296 who underwent OPD). There was no significant difference in the number of harvested lymph nodes between the two groups (MD 0.98; 95\% CI -1.32 to $3.27 ; P=0.4$ ) (Fig. 4 C). The heterogeneity was high $\left(I^{2}=86 \%\right)$, and a random effects model was adopted. Moreover, data regarding lymph node positivity were also reported in these studies; the pooled results showed no statistical difference between the two groups (MD $=0.96$; $95 \%$ CI $0.74-1.26 ; P=0.78, I^{2}=92 \%$ ) (Fig. 4D). Our sensitivity analysis showed no obvious differences.

\section{Time to starting adjuvant chemotherapy after surgery}

Four studies [15, 20-22] gathered data on the time to starting adjuvant chemotherapy after surgery, including a total of 10,494 patients (1242 and 9252 underwent MIPD and OPD, respectively). The pooled results indicated that the time to adjuvant chemotherapy was significantly shorter in the MIPD group (MD $=-15.35 ; 95 \% \mathrm{CI}-30.33$ to -0.36 ; $P=0.04)$ (Fig. 4E). The heterogeneity was high $\left(I^{2}=98 \%\right)$, and a random effects model was adopted.

\section{OS and DFS}

DFS data were available in 2 studies [15, 16]. A significantly longer DFS was observed in the MIPD group (HR 1.30, 95\% CI 1.02 to $1.66, P=0.04, I^{2}=0 \%$ ) (Fig. 5A). Eight studies $[15,16,18-23]$ investigated OS; their pooled data revealed no significant difference between patients who underwent MIPD and those who underwent OPD (HR 0.98, 95\% CI 0.95 to $1.02, P=0.36, I^{2}=0 \%$ ) (Fig. $5 \mathrm{~B}$ ).

\section{Publication bias}

Begg's funnel plot was used to assess any publication bias present in the articles. As shown in the funnel plot of OS (Fig. 6), no evidence of significant publication bias was found.

\section{Discussion}

To our knowledge, this is the first meta-analysis designed to specifically evaluate the perioperative and oncological outcomes of patients with PDAC who underwent MIPD and compare the findings with those for patients who underwent OPD. Overall, the pooled results revealed no significant difference in OS between the two groups, although patients who underwent MIPD had longer DFS periods and 


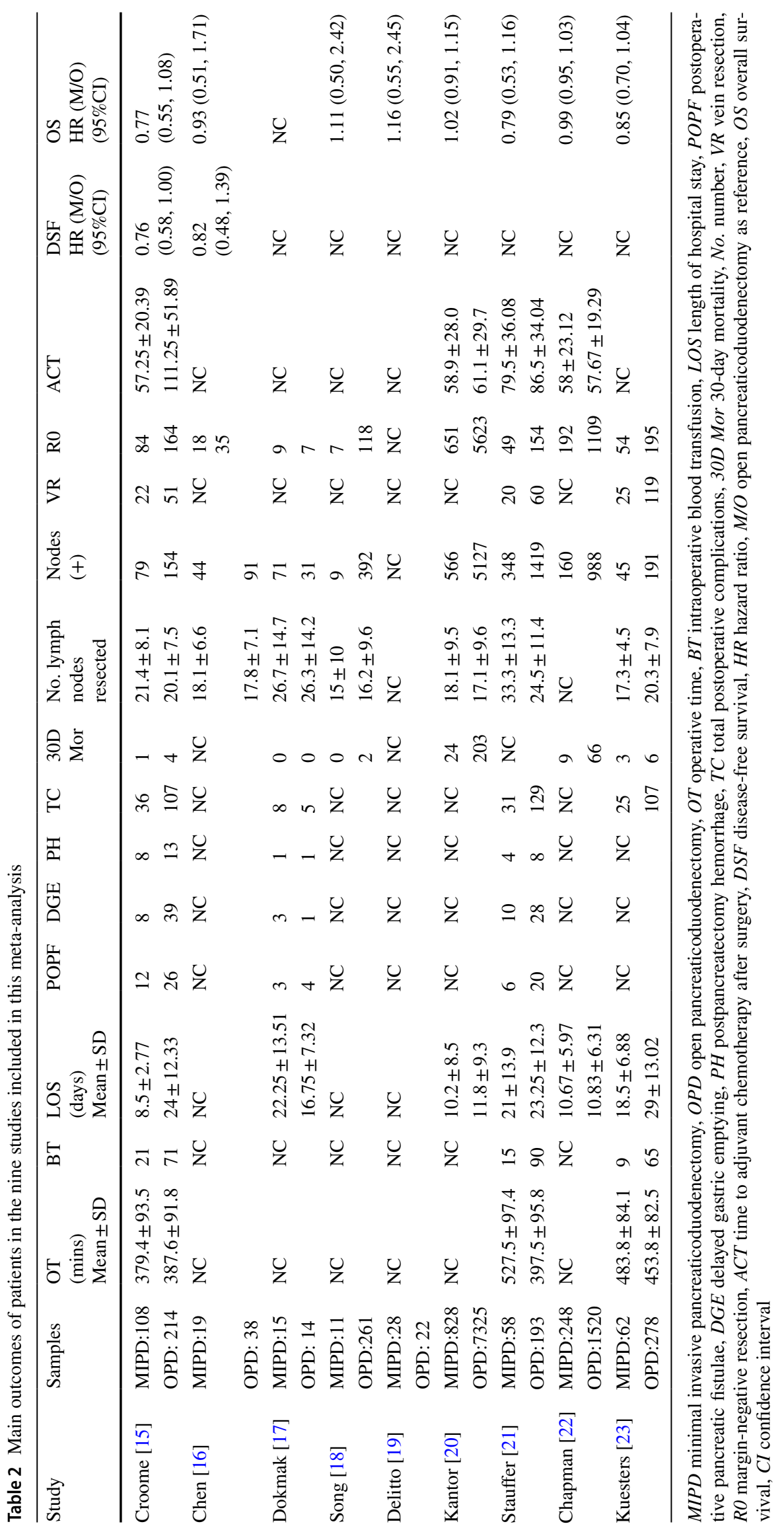



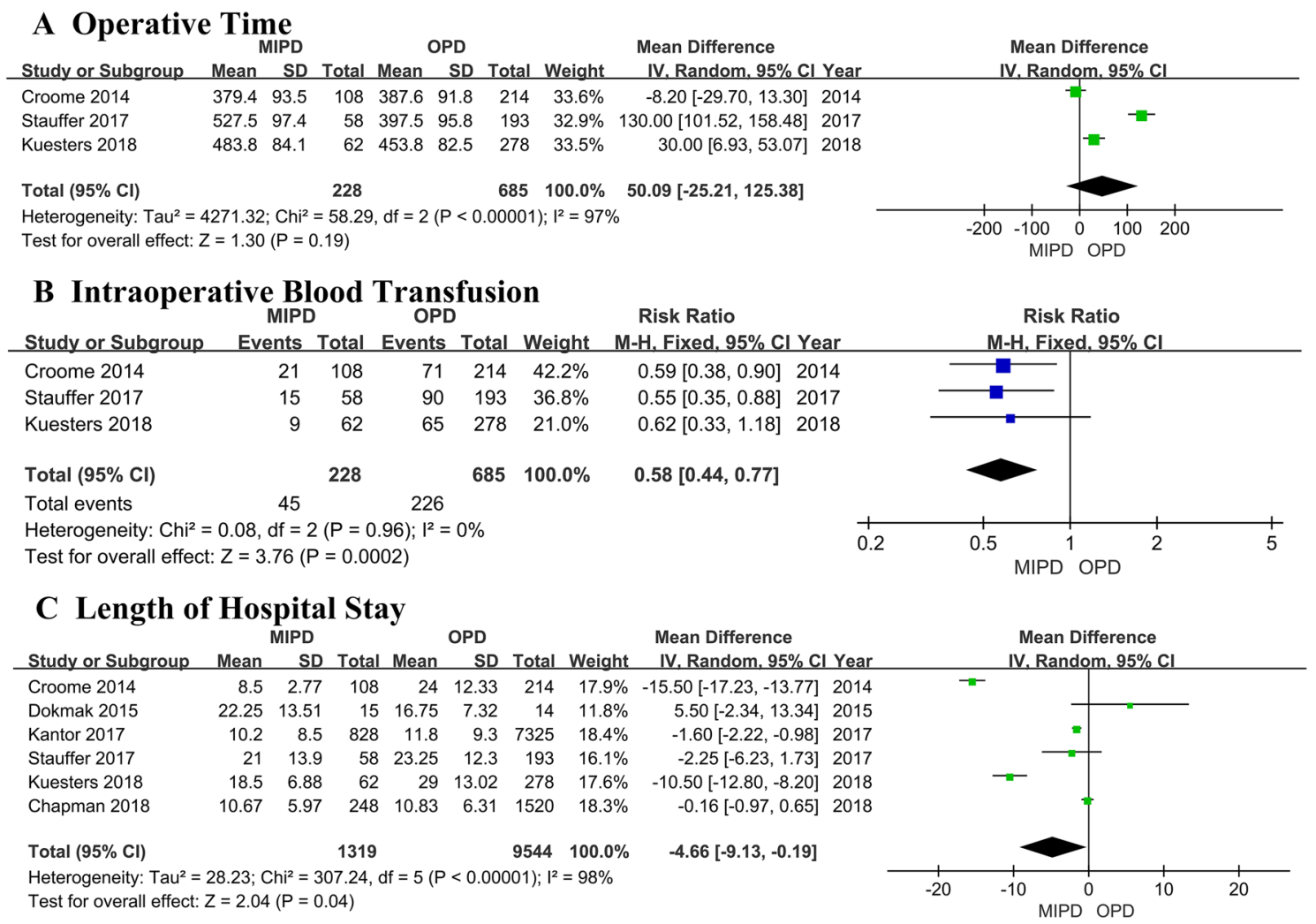

Fig. 2 Comparison of perioperative outcomes between patients who underwent minimally invasive pancreaticoduodenectomy (MIPD) and those who underwent open pancreaticoduodenectomy (OPD) for pancreatic duct adenocarcinoma

commenced adjuvant chemotherapy sooner after surgery. Moreover, we found no significant difference in operative time, postoperative complications, 30-day mortality, vein resection, number of harvested lymph nodes, or number of positive lymph nodes between the two approaches. There was a significant improvement in margin-negative resection with MIPD, as well as a shorter LOS and a lower intraoperative blood transfusion volume. Thus, our findings suggest that the outcomes of MIPD are at least equivalent to, if not better than, those of OPD in patients with PDAC.

It was previously thought that operating time would be longer with MIPD; this method is certainly challenging and has a steep learning curve. A recent retrospective multicenter analysis by Wang et al. [24] found that the learning curve for MIPD had 3 phases, with peaks evident after the completion of 40 and 104 cases. They also concluded that the operative time significantly decreased from phase I to phase III and was even comparable with that of OPD, consistent with the findings of van Hilst et al. [25] and Boone et al. [26]. In the present study, no significant difference between the 2 approaches was observed in terms of operative time. Croome et al. [15] excluded 10 patients who underwent MIPD in the first 6 months to avoid the early segment of their learning curve, which may have introduced bias with regard to our pooled results; however, we found no significant change in our results when excluding their study from our analysis. Therefore, our findings suggest that the operating time for MIPD is comparable to that of OPD once the learning curve is achieved.

The feasibility and safety of MIPD have been established given that enthusiasm for this procedure continues to grow quickly. MIPD has the potential to markedly reduce intraoperative blood loss and transfusion, as well as LOS; these attributes were corroborated in our own study. Although there were no sufficient data for analyzing intraoperative blood loss, the rate of blood transfusion was lower in patients undergoing MIPD in the present study, which ought to reflect lower intraoperative blood loss. Allogeneic blood transfusion has been confirmed to be an independent negative predictor of DFS and OS among patients with PDAC who underwent PD $[27,28]$. Therefore, MIPD may potentially be related to improved survival compared to OPD in patients with this disease. A shorter LOS could also translate into faster recovery, which may help introduce subsequent treatments sooner. LOS is also an important component of healthcare costs [29], and whether opting for MIPD can significantly reduce such costs over OPD is an important area of active investigation [30]. 


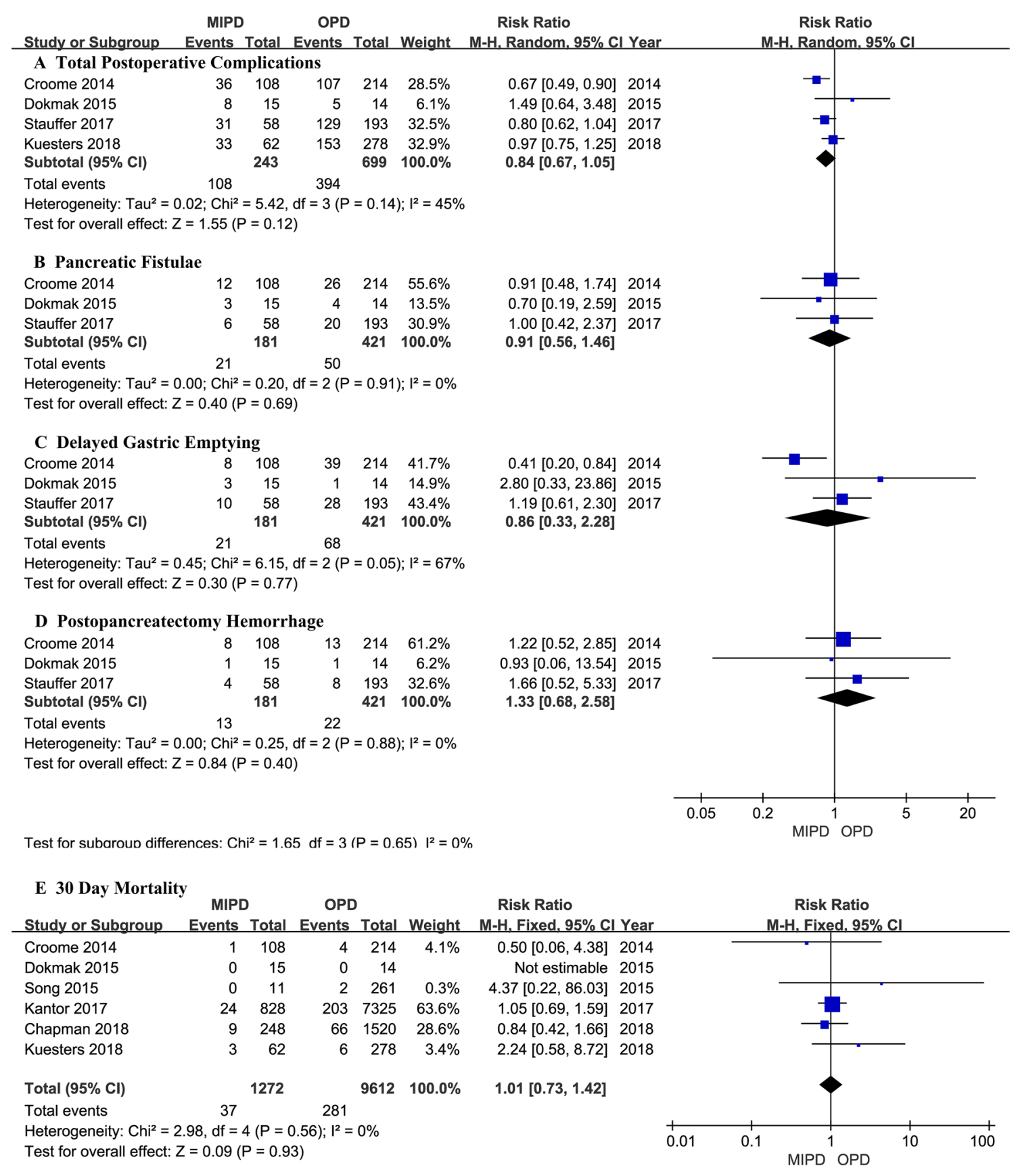

Fig. 3 Comparison of postoperative and 30-day mortality between patients who underwent minimally invasive pancreaticoduodenectomy (MIPD) and those who underwent open pancreaticoduodenectomy (OPD) for pancreatic duct adenocarcinoma

In the present study, total and major complications attributed to MIPD occurred at a similar rate to those attributed to OPD, including postoperative pancreatic fistulae, delayed gastric emptying, postpancreatectomy hemorrhage, and 30-day mortality. Other studies have also observed such outcomes [25, 31, 32]. Therefore, the minimally invasive approach does not appear to alter the risk of severe complications and death compared to OPD. This is highly important because most resections are performed to treat malignant diseases. Moreover, Stauffer et al. showed that major complications may be an independent predictor of poorer survival [21]. It is highly probable that major postoperative complications would at least delay the administration of subsequent chemotherapy or limit the patient's ability to tolerate a full course.

Our study also found that surrogate oncologic indicators including the number of resected veins, number of harvested lymph nodes, and rate of positive nodes were similar in the 


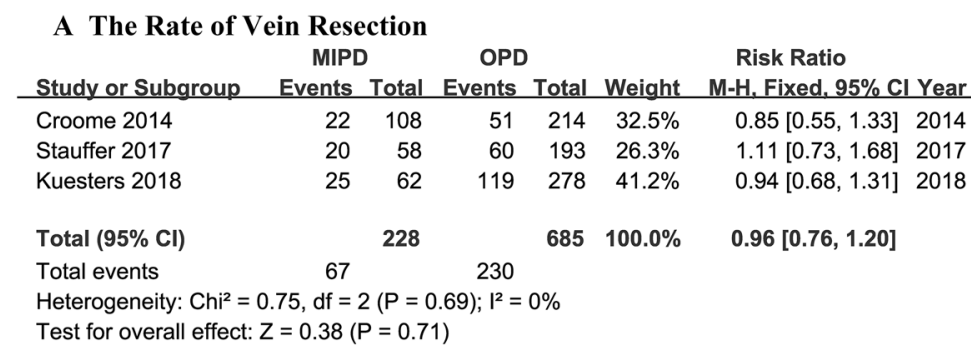

\begin{tabular}{|c|c|c|c|c|c|c|c|}
\hline & MIPD & & OPD & & & Risk Ratio & \\
\hline tudy or Subgroup & Event: & Total & Even & Total & Weight & M-H, Random, 95\% & Yea \\
\hline Croome 2014 & 84 & 108 & 164 & 214 & $11.4 \%$ & $1.01[0.90,1.15]$ & 2014 \\
\hline Chen 2015 & 18 & 19 & 35 & 38 & $9.5 \%$ & $1.03[0.89,1.18]$ & 2015 \\
\hline Song 2015 & 7 & 11 & 118 & 261 & $1.1 \%$ & $1.41[0.88,2.24]$ & 2015 \\
\hline Dokmak 2015 & 9 & 15 & 7 & 14 & $0.5 \%$ & $1.20[0.62,2.34]$ & 2015 \\
\hline Kantor 2017 & 651 & 828 & 5623 & 7325 & $33.4 \%$ & $1.02[0.99,1.06]$ & 2017 \\
\hline Stauffer 2017 & 49 & 58 & 154 & 193 & $10.6 \%$ & $1.06[0.93,1.21]$ & 2017 \\
\hline Chapman 2018 & 192 & 248 & 1109 & 1520 & $21.6 \%$ & $1.06[0.99,1.14]$ & 2018 \\
\hline Kuesters 2018 & 54 & 62 & 195 & 278 & $11.7 \%$ & $1.24[1.10,1.40]$ & 2018 \\
\hline Total $(95 \% \mathrm{Cl})$ & & 1349 & & 9843 & $100.0 \%$ & $1.06[1.01,1.12]$ & \\
\hline Total events & 1064 & & 7405 & & & & \\
\hline $\begin{array}{l}\text { Heterogeneity: Tau } \\
\text { Test for overall effe }\end{array}$ & $\begin{array}{l}0 ; \mathrm{Ch}^{2} \\
2.43(\mathrm{~F}\end{array}$ & $\begin{array}{l}\text { D } 10.8 \\
\partial=0.0\end{array}$ & df = 7 & & 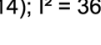 & & \\
\hline
\end{tabular}

\begin{tabular}{|c|c|c|c|c|c|c|c|}
\hline \multicolumn{4}{|c|}{ C Harvested Lymph Nodes } & \multicolumn{2}{|c|}{ OPD } & \multirow[b]{2}{*}{ Total } & \multirow[b]{2}{*}{ Weight } \\
\hline Study or Subgroup & Mean & SD & Total & Mean & SD & & \\
\hline Croome 2014 & 21.4 & 8.1 & 108 & 20.1 & 7.5 & 214 & $19.1 \%$ \\
\hline Dokmak 2015 & 26.7 & 14.7 & 15 & 26.3 & 14.2 & 14 & $3.9 \%$ \\
\hline Song 2015 & 15 & 10 & 11 & 16.2 & 9.6 & 261 & $8.7 \%$ \\
\hline Chen 2015 & 18.1 & 6.6 & 19 & 17.8 & 7.1 & 38 & $13.8 \%$ \\
\hline Stauffer 2017 & 33.3 & 13.3 & 54 & 24.5 & 11.4 & 166 & $13.2 \%$ \\
\hline Kantor 2017 & 18.1 & 9.5 & 828 & 17.1 & 9.6 & 7325 & $21.3 \%$ \\
\hline Kuesters 2018 & 17.3 & 4.5 & 62 & 20.3 & 7.9 & 278 & $20.0 \%$ \\
\hline
\end{tabular}

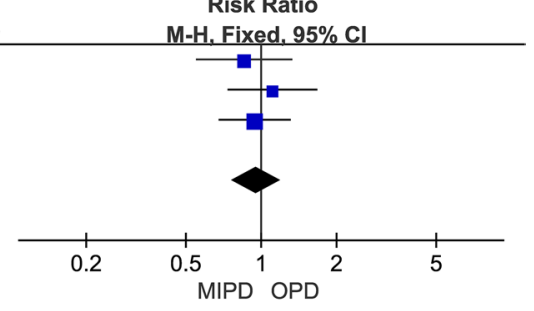

M-H. Fixed, $95 \%$ C

\footnotetext{
$\begin{array}{llll}\text { Total }(95 \% \mathrm{Cl}) & 1097 & 8296 & 100.0 \%\end{array}$

Heterogeneity: $\mathrm{Tau}^{2}=6.32 ; \mathrm{Chi}^{2}=42.29, \mathrm{df}=6(\mathrm{P}<0.00001) ; \mathrm{I}^{2}=86 \%$

Test for overall effect: $Z=0.83(P=0.40)$
}

IV. Random, $95 \%$ Cl Year

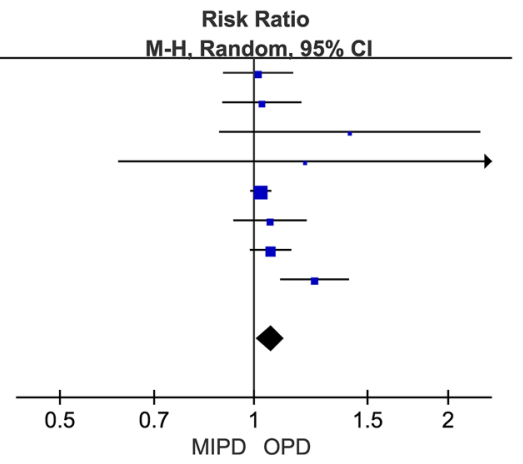

Risk Ratio

D Positive Lymph Nodes
MIPD $\begin{gathered}\text { OPD } \\ \text { Study or Subgroup Events Total Events }\end{gathered}$

$154 \quad 4301 \quad 152 \% \quad 0.95[0,73,1,25] 2014$

$\begin{array}{llllllll}\text { Croome } 2014 & 79 & 2311 & 154 & 4301 & 15.2 \% & 0.95[0.73,1,25] & 2014\end{array}$

$\begin{array}{lrrrrr}\text { Song } 2015 & 9 & 165 & 392 & 4228 & 8.8 \%\end{array}$

$\begin{array}{llllll}\text { Dokmak } 2015 & 71 & 401 & 31 & 368 & 12.8 \%\end{array}$

$\begin{array}{llllll}\text { Chen } 2015 & 44 & 344 & 91 & 676 & 13.9 \%\end{array}$

$\begin{array}{llllll}\text { Stauffer } 2017 & 348 & 1931 & 1419 & 4729 & 17.4 \%\end{array}$

$\begin{array}{llllll}\text { Kantor } 2017 & 566 & 14987 & 5127 & 125258 & 17.6 \%\end{array}$

$\begin{array}{llllrl}\text { Kuesters } 2018 & 45 & 1073 & 191 & 5643 & 14.3 \%\end{array}$

$1.30[-0.53,3.13] 2014$

$-1.20[-7.22,4.82] 2015$

$0.30[-3.43,4.03] 2015$

$8.80[4.85,12.75] 2017$

$1.00[0.32,1.68] 2017$

$-3.00[-4.46,-1.54] 2018$

Total $(95 \% \mathrm{Cl})$

21212

Total events

1162

$0.59[0.31,1.12] 2015$

$2.10[1.41,3.13] 2015$

$0.95[0.68,1.33] 2015$

$0.60[0.54,0.67] 2017$

$0.92[0.85,1.00] 2017$

$1.24[0.90,1.70] 2018$

Heterogeneity: $\mathrm{Tau}^{2}=0.10 ; \mathrm{Chi}^{2}=72.47, \mathrm{df}=6(\mathrm{P}<0.00001) ; \mathrm{I}^{2}=92 \%$

$0.96[0.74,1.26]$

Test for overall effect: $Z=0.28(P=0.78)$

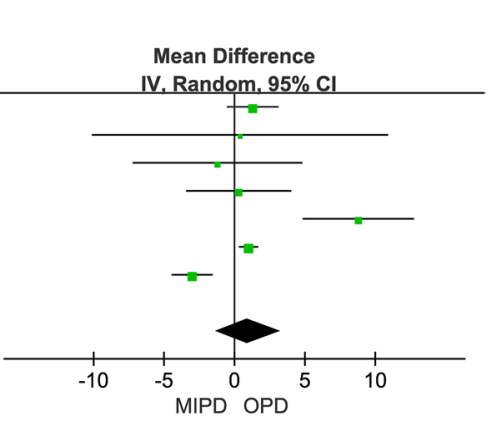

\section{E Time to Adjuvant Chemotherapy After Surgery}

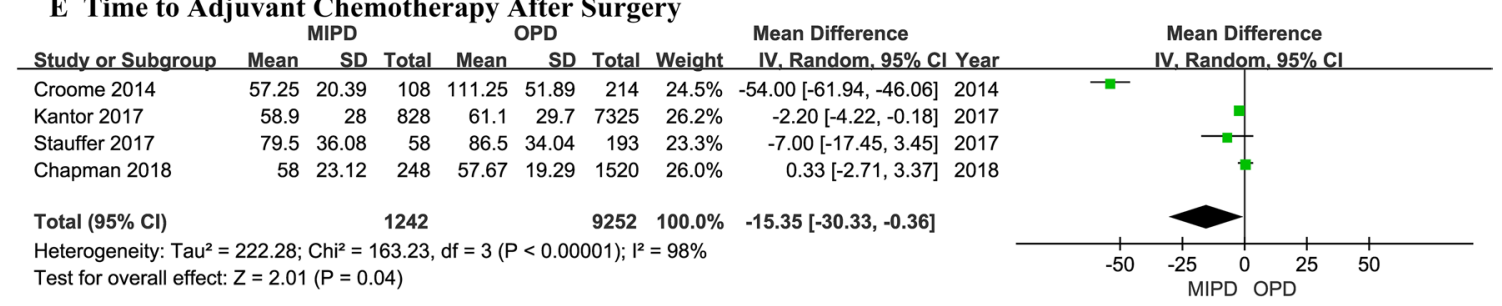

Fig. 4 Short-term oncological outcomes of patients who underwent minimally invasive pancreaticoduodenectomy (MIPD) and those who underwent open pancreaticoduodenectomy (OPD) for pancreatic duct adenocarcinoma 


\section{A Disease-Free Survival}

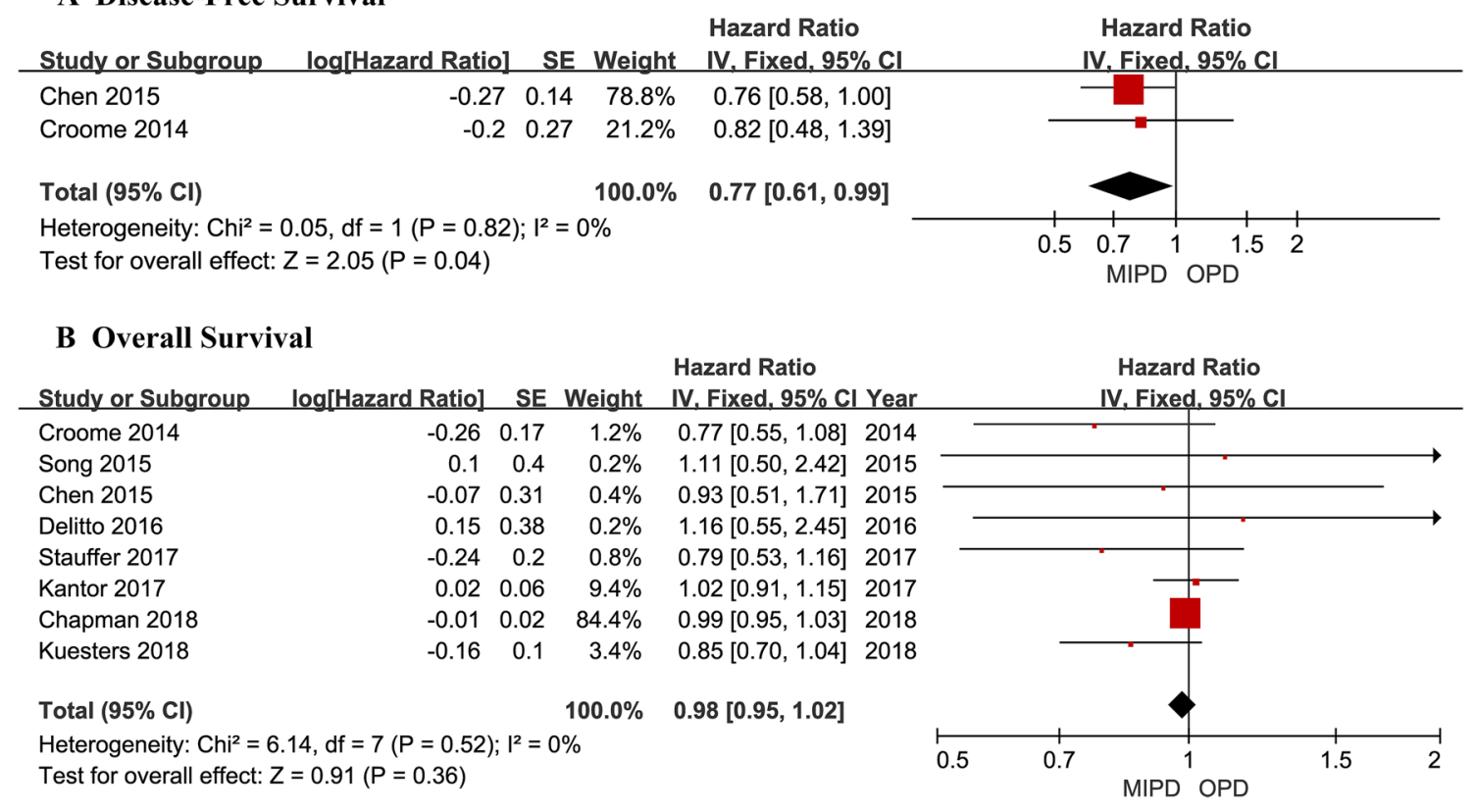

Fig. 5 Long-term oncological outcomes of patients who underwent minimally invasive pancreaticoduodenectomy (MIPD) and those who underwent open pancreaticoduodenectomy (OPD) for pancreatic duct adenocarcinoma

Fig. 6 Begg's funnel plot for assessing publication bias

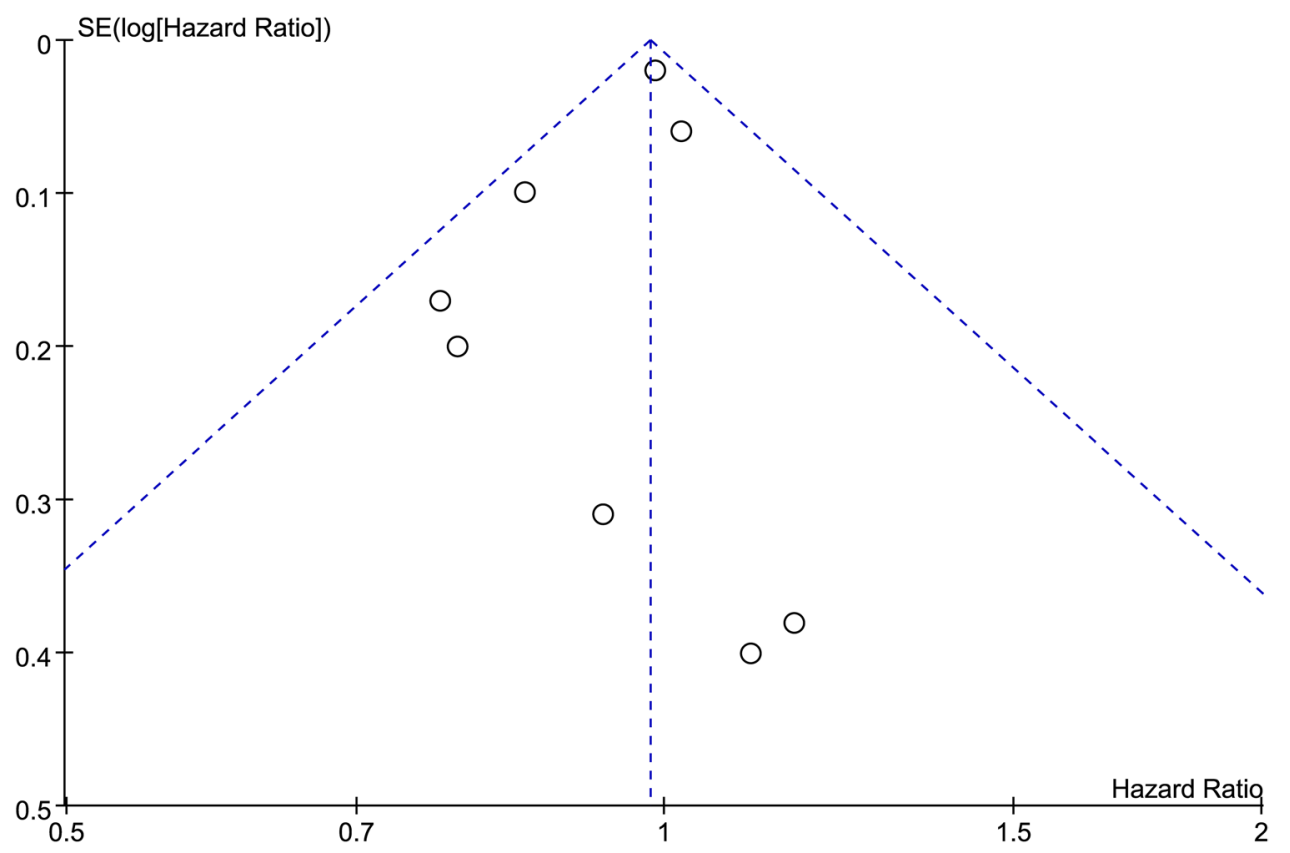

2 groups, although the rate of $\mathrm{R} 0$ resection was higher in the MIPD group than in the OPD group. MIPD was contraindicated in patients who required vascular resection and reconstruction in a previous study [33]. However, Croome et al. [34] suggested that MIPD with major vascular resection is feasible and safe, and it can achieve similar results in terms of morbidity and mortality rates as well as of oncologic outcomes as does OPD with major vascular resection. The latter notion is supported by our data. Nevertheless, the acquisition of considerable experience for performing OPD both with and without vascular resection and for performing MIPD without vascular resection is strongly recommended before attempting minimally invasive major vein resection and reconstruction [35]. Previous studies found that the lymph node and margin statuses were significant predictors of DFS and OS [21, 36]. Our pooled results revealed that MIPD 
produced oncologic outcomes similar to, or even more favorable than, OPD did. Moreover, Stauffer et al. [21] found that the proportion of positive lymph nodes in patients who underwent MIPD was lower than that in patients who underwent OPD, and that the negative margin rate was higher; however, neither finding was statistically significant. These results may indicate that the minimally invasive approach allows for complete and adequate lymphadenectomy and pancreatic resection as it provides superior and magnified views of the tumor.

The primary results of this study showed a significant improvement in the time to starting adjuvant chemotherapy and in DFS among patients with PDAC who underwent MIPD; Conrad et al. [31] reported similar outcomes in their study. According to a recent evaluation of the European Study Group for Pancreatic Cancer, completion of all 6 cycles of adjuvant chemotherapy following resection of PDAC improves OS if the chemotherapy is started within 12 weeks [37]. Croome et al. [15] reported that the lack of chemotherapy within 90 days of surgery was a strong predictor of poorer OS; this suggests that the inability to initiate or complete chemotherapy in a timely manner will ultimately have a negative influence on survival. Our data do indicate a longer DFS in the MIPD group than in the OPD group, which may reflect the difference in the timing of adjuvant treatment initiation. Therefore, we posit that MIPD may enhance the ability of patients to receive subsequent treatments in a timely fashion after surgery and to complete their dosing schedules as well.

Another primary result in our study was the lack of a significant difference in OS between the 2 approaches. Chapman et al. [22] reported that patients undergoing MIPD had a noticeably longer median survival time than did those undergoing OPD (19.8 vs. 15.6 months) as well as an improved OS (HR $0.85,95 \%$ CI 0.69 to 10.3 ) after adjusting for patient- and tumor-related characteristics; these findings were similar to those of Croome et al. [15] and Conrad et al. [31]. Furthermore, Stauffer et al. [21] also found that the 5-year survival rates of patients who underwent MIPD and OPD were $32 \%$ and $15.34 \%$, respectively. However, the differences in OS in these aforementioned studies were not significant. It remains to be determined whether MIPD can result in a significantly different OS among patients with PDCA as additional patients are accrued and follow-up periods are lengthened. Nevertheless, in our opinion, one of the key objectives for achieving superior oncologic outcomes with existing treatment modalities is to improve the ability of patients to receive both complete tumor resection and chemotherapy, and we believe that MIPD is a realistic method to meet this objective.

Two other meta-analyses that were similar to ours were identified in our search $[8,38]$. Although the stated aims of both were to assess LPD versus OPD in patients with
PDAC, they included patients with ampullary carcinoma, some benign tumors, and even chronic pancreatitis in addition to those with PDACs. Moreover, the pooled results of short-term oncological outcomes such as R0 resection and number of lymph nodes retrieved were different from ours, since we only included patients with PDAC. Even though their findings regarding OS were similar to ours, they found 4 of the studies that were also included in our analysis [15, 20-22] to be heterogeneous (although we did not), whereas 8 other studies were not heterogeneous in their analyses (as consistent with our data). Consequently, their meta-analyses may prevent achieving objective conclusions regarding the oncologic safety of MIPD.

There were some limitations to our study that should be taken into account when considering the results. First, all included studies were retrospective and conducted in high-volume hospitals. Numerous studies have shown that hospital volume is significantly correlated with the incidence of perioperative outcomes [24]. Kantro et al. [20] reported that the 30-day mortality rate for patients who underwent MIPD was higher in low-volume hospitals than in high-volume institutions; therefore, there was a risk of selection bias even though such confounders could not be avoided. Second, the numbers of patients in some of the studies were too small, leading to low-power analyses. Third, only one of the included studies [16] compared RPD and OPD, which may have introduced bias because robotic and laparoscopic approaches have substantial differences, and the number of patients who underwent RPD was small and accounted for a small proportion in the analysis of results regarding the $\mathrm{R} 0$ resection rate, number of harvested lymph nodes, rate of positive nodes, and OS. However, the results and heterogeneity for all these variables showed no significant change after exclusion of the study comparing RPD and OPD. Nevertheless, some of our conclusions, particularly those pertaining to RPD, should be interpreted with caution. In the future, when more data are available, subgroup analysis should be performed to determine whether is any difference between the laparoscopic and robotic approaches. Finally, the heterogeneity in some of the results was high. Although we conducted sensitivity analyses, the results and heterogeneity were barely changed. Thus, some of our results should be interpreted with caution. Overall, additional prospective and multicenter randomized controlled trials with longer follow-up periods are warranted to compare the oncological outcomes of MIPD and OPD.

In conclusion, the results of our meta-analysis indicate that MIPD appears to be safe and feasible with perioperative, short-term, and long-term outcomes that are similar to those of OPD in the setting of PDAC. Moreover, MIPD may also provide advantages such as lower transfusion rates, higher rates of margin-negative resection, shorter time to starting 
adjuvant chemotherapy, and longer DFS when compared to OPD in patients with PDAC.

Acknowledgements We thank the authors who provided us with the full text and relevant data from their studies. We would like to thank Editage (www.editage.cn) for English language editing. Rui Sun and Jiawen Yu contributed to the conception and design, acquisition, analysis, and interpretation, drafted manuscript, critically revised manuscript. Rui Sun and Jiawen Yu contributed equally to this article. Yifan Zhang and Zhikai Liang contributed to analysis. Xianlin Han critically reviewed the manuscript. All the authors approved the final draft.

Funding This meta-analysis is supported by the Science Fund for Youth in Peking Union Medical College (No. 2017310004).

\section{Compliance with ethical standards}

Disclosures Xianlin Han, Rui Sun, Jiawen Yu, Yifan Zhang, and Zhikai Liang have no conflicts of interest or financial ties to disclose.

Open Access This article is licensed under a Creative Commons Attribution 4.0 International License, which permits use, sharing, adaptation, distribution and reproduction in any medium or format, as long as you give appropriate credit to the original author(s) and the source, provide a link to the Creative Commons licence, and indicate if changes were made. The images or other third party material in this article are included in the article's Creative Commons licence, unless indicated otherwise in a credit line to the material. If material is not included in the article's Creative Commons licence and your intended use is not permitted by statutory regulation or exceeds the permitted use, you will need to obtain permission directly from the copyright holder. To view a copy of this licence, visit http://creativecommons.org/licenses/by/4.0/.

\section{References}

1. Bray F, Ferlay J, Soerjomataram I, Siegel RL, Torre LA, Jemal A (2018) Global cancer statistics 2018: GLOBOCAN estimates of incidence and mortality worldwide for 36 cancers in 185 countries. CA Cancer J Clin 68:394-424

2. Gagner M, Pomp A (1994) Laparoscopic pylorus-preserving pancreatoduodenectomy. Surg Endosc 8:408-410

3. Giulianotti PC, Coratti A, Angelini M, Sbrana F, Cecconi S, Balestracci T, Caravaglios G (2003) Robotics in general surgery: personal experience in a large community hospital. Arch Surg 138:777-784

4. Doula C, Kostakis ID, Damaskos C, Machairas N, Vardakostas DV, Feretis T, Felekouras E (2016) Comparison between minimally invasive and open pancreaticoduodenectomy: a systematic review. Surg Laparosc Endosc Percutan Tech 26:6-16

5. Chopinet S, Fuks D, Rinaudo M, Massol J, Gregoire E, Lamer C, Belgaumkar A, Hardwigsen J, Le Treut YP, Gayet B (2018) Postoperative bleeding after laparoscopic pancreaticoduodenectomy: the Achilles' Heel? World J Surg 42:1138-1146

6. Correa-Gallego C, Dinkelspiel HE, Sulimanoff I, Fisher S, Vinuela EF, Kingham TP, Fong Y, DeMatteo RP, D'Angelica MI, Jarnagin WR, Allen PJ (2014) Minimally-invasive vs open pancreaticoduodenectomy: systematic review and meta-analysis. J Am Coll Surg 218:129-139

7. Chen K, Liu XL, Pan Y, Maher H, Wang XF (2018) Expanding laparoscopic pancreaticoduodenectomy to pancreatic-head and periampullary malignancy: major findings based on systematic review and meta-analysis. BMC Gastroenterol 18:102

8. Jiang YL, Zhang RC, Zhou YC (2019) Comparison of overall survival and perioperative outcomes of laparoscopic pancreaticoduodenectomy and open pancreaticoduodenectomy for pancreatic ductal adenocarcinoma: a systematic review and meta-analysis. BMC Cancer 19:781

9. Yin Z, Jian Z, Hou B, Jin H (2019) Surgical and oncological outcomes of laparoscopic versus open pancreaticoduodenectomy in patients with pancreatic duct adenocarcinoma. Pancreas 48:861-867

10. Moher D, Liberati A, Tetzlaff J, Altman DG (2010) Preferred reporting items for systematic reviews and meta-analyses: the PRISMA statement. Int J Surg 8:336-341

11. Stang A (2010) Critical evaluation of the Newcastle-Ottawa scale for the assessment of the quality of nonrandomized studies in meta-analyses. Eur J Epidemiol 25:603-605

12. Wan X, Wang W, Liu J, Tong T (2014) Estimating the sample mean and standard deviation from the sample size, median, range and/or interquartile range. BMC Med Res Methodol 14:135

13. Luo D, Wan X, Liu J, Tong T (2018) Optimally estimating the sample mean from the sample size, median, mid-range, and/or mid-quartile range. Stat Methods Med Res 27:1785-1805

14. Tierney JF, Stewart LA, Ghersi D, Burdett S, Sydes MR (2007) Practical methods for incorporating summary time-to-event data into meta-analysis. Trials 8:16

15. Croome KP, Farnell MB, Que FG, ReidLombardo K, Truty MJ, Nagorney DM, Kendrick ML (2014) Total laparoscopic pancreaticoduodenectomy for pancreatic ductal adenocarcinoma oncologic advantages over open approaches? Ann Surg 260:633-640

16. Chen S, Chen JZ, Zhan Q, Deng XX, Shen BY, Peng CH, Li HW (2015) Robot-assisted laparoscopic versus open pancreaticoduodenectomy: a prospective, matched, mid-term follow-up study. Surg Endosc 29:3698-3711

17. Dokmak S, Fteriche FS, Aussilhou B, Bensafta Y, Levy P, Ruszniewski P, Belghiti J, Sauvanet A (2015) Laparoscopic pancreaticoduodenectomy should not be routine for resection of periampullary tumors. J Am Coll Surg 220:831-838

18. Song KB, Kim SC, Hwang DW, Lee JH, Lee DJ, Lee JW, Park KM, Lee YJ (2015) Matched case-control analysis comparing laparoscopic and open pylorus-preserving pancreaticoduodenectomy in patients with periampullary tumors. Ann Surg 262:146-155

19. Delitto D, Luckhurst CM, Black BS, Beck JL, George TJ, Sarosi GA, Thomas RM, Trevino JG, Behrns KE, Hughes SJ (2016) Oncologic and perioperative outcomes following selective application of laparoscopic pancreaticoduodenectomy for periampullary malignancies. J Gastrointest Surg 20:1343-1349

20. Kantor O, Talamonti MS, Sharpe S, Lutfi W, Winchester DJ, Roggin KK, Bentrem DJ, Prinz RA, Baker MS (2017) Laparoscopic pancreaticoduodenectomy for adenocarcinoma provides shortterm oncologic outcomes and long-term overall survival rates similar to those for open pancreaticoduodenectomy. Am J Surg 213:512-515

21. Stauffer JA, Coppola A, Villacreses D, Mody K, Johnson E, Li Z, Asbun HJ (2017) Laparoscopic versus open pancreaticoduodenectomy for pancreatic adenocarcinoma: long-term results at a single institution. Surg Endosc 31:2233-2241

22. Chapman BC, Gajdos C, Hosokawa P, Henderson W, Paniccia A, Overbey DM, Gleisner A, Schulick RD, McCarter MD, Edil BH (2018) Comparison of laparoscopic to open pancreaticoduodenectomy in elderly patients with pancreatic adenocarcinoma. Surg Endosc 32:2239-2248

23. Kuesters S, Chikhladze S, Makowiec F, Sick O, FichtnerFeigl S, Hopt UT, Wittel UA (2018) Oncological outcome of 
laparoscopically assisted pancreatoduodenectomy for ductal adenocarcinoma in a retrospective cohort study. Int J Surg 55:162-166

24. Wang M, Peng B, Liu J, Yin X, Tan Z, Liu R, Hong D, Zhao W, Wu H, Chen R, Li D, Huang H, Miao Y, Liu Y, Liang T, Wang W, Cai Y, Xing Z, Cheng W, Zhong X, Zhao Z, Zhang J, Yang Z, Li G, Shao Y, Lin G, Jiang K, Wu P, Jia B, Ma T, Jiang C, Peng S (2019) Practice patterns and perioperative outcomes of laparoscopic pancreaticoduodenectomy in china: a retrospective multicenter analysis of 1029 patients. Ann Surg. https://doi. org/10.1097/SLA.0000000000003190

25. van Hilst J, Bosscha K, Brinkman DJ, van Dieren S, Dijkgraaf MG, Gerhards MF, de Hingh IH, Karsten TM, Lips DJ, Luyer MD, Busch OR, Festen S, Besselink MG (2019) Laparoscopic versus open pancreatoduodenectomy for pancreatic or periampullary tumours (LEOPARD-2): a multicentre, patient-blinded, randomised controlled phase 2/3 trial. Lancet Gastroenterol Hepatol 4:199-207

26. Boone BA, Zenati M, Hogg ME, Steve J, Moser AJ, Bartlett DL, Zeh HJ, Zureikat AH (2015) Assessment of quality outcomes for robotic pancreaticoduodenectomy: identification of the learning curve. JAMA Surg 150:416-422

27. Kneuertz PJ, Patel SH, Chu CK, Maithel SK, Sarmiento JM, Delman KA, Staley CA 3rd, Kooby DA (2011) Effects of perioperative red blood cell transfusion on disease recurrence and survival after pancreaticoduodenectomy for ductal adenocarcinoma. Ann Surg Oncol 18:1327-1334

28. Sutton JM, Kooby DA, Wilson GC, Squires MH 3rd, Hanseman DJ, Maithel SK, Bentrem DJ, Weber SM, Cho CS, Winslow ER, Scoggins CR, Martin RC 2nd, Kim HJ, Baker JJ, Merchant NB, Parikh AA, Abbott DE, Edwards MJ, Ahmad SA (2014) Perioperative blood transfusion is associated with decreased survival in patients undergoing pancreaticoduodenectomy for pancreatic adenocarcinoma: a multi-institutional study. J Gastrointest Surg 18:1575-1587

29. Mirkin KA, Greenleaf EK, Hollenbeak CS, Wong J (2018) Minimally invasive surgical approaches offer earlier time to adjuvant chemotherapy but not improved survival in resected pancreatic cancer. Surg Endosc 32:2387-2396

30. Kutlu OC, Lee JE, Katz MH, Tzeng CWD, Wolff RA, Varadhachary GR, Vauthey JN, Fleming JB, Conrad C (2018) Open pancreaticoduodenectomy case volume predicts outcome of laparoscopic approach: a population-based analysis. Am J Surg 267:552-560

31. Conrad C, Basso V, Passot G, Zorzi D, Li L, Chen HC, Fuks D, Gayet B (2017) Comparable long-term oncologic outcomes of laparoscopic versus open pancreaticoduodenectomy for adenocarcinoma: a propensity score weighting analysis. Surg Endosc 31:3970-3978

32. Kantor O, Pitt HA, Talamonti MS, Roggin KK, Bentrem DJ, Prinz RA, Baker MS (2018) Minimally invasive pancreatoduodenectomy: is the incidence of clinically relevant postoperative pancreatic fistula comparable to that after open pancreatoduodenectomy? Surg 163:587-593

33. Asbun HJ, Stauffer JA (2012) Laparoscopic vs open pancreaticoduodenectomy: overall outcomes and severity of complications using the accordion severity grading system. J Am Coll Surg 215:810-819

34. Croome KP, Farnell MB, Que FG, Reid-Lombardo KM, Truty MJ, Nagorney DM, Kendrick ML (2015) Pancreaticoduodenectomy with major vascular resection: a comparison of laparoscopic versus open approaches. J Gastrointest Surg 19:189-194

35. Rebelo A, Michalski CW, Ukkat J, Kleeff J (2019) Pancreatic cancer surgery with vascular resection. J Pancreato $2: 1-5$

36. Neoptolemos JP, Stocken DD, Bassi C, Ghaneh P, Cunningham D, Goldstein D, Padbury R, Moore MJ, Gallinger S, Mariette C, Wente MN, Izbicki JR, Friess H, Lerch MM, Dervenis C, Olah A, Butturini G, Doi R, Lind PA, Smith D, Valle JW, Palmer DH, Buckels JA, Thompson J, McKay CJ, Rawcliffe CL, Buchler MW (2010) Adjuvant chemotherapy with fluorouracil plus folinic acid vs gemcitabine following pancreatic cancer resection: a randomized controlled trial. JAMA 304:1073-1081

37. Neoptolemos JP, Palmer DH, Ghaneh P, Psarelli EE, Valle JW, Halloran CM, Faluyi O, O'Reilly DA, Cunningham D, Wadsley J, Darby S, Meyer T, Gillmore R, Anthoney A, Lind P, Glimelius B, Falk S, Izbicki JR, Middleton GW, Cummins S, Ross PJ, Wasan H, McDonald A, Crosby T, Ma YT, Patel K, Sherriff D, Soomal R, Borg D, Sothi S, Hammel P, Hackert T, Jackson R, Buchler MW (2017) Comparison of adjuvant gemcitabine and capecitabine with gemcitabine monotherapy in patients with resected pancreatic cancer (ESPAC-4): a multicentre, open-label, randomised, phase 3 trial. Lancet 389:1011-1024

38. Peng L, Zhou Z, Cao Z, Wu W, Xiao W, Cao J (2019) Longterm oncological outcomes in laparoscopic versus open pancreaticoduodenectomy for pancreatic cancer: a systematic review and meta-analysis. J Laparoendosc Adv Surg Tech A 29:759-769

Publisher's Note Springer Nature remains neutral with regard to jurisdictional claims in published maps and institutional affiliations. 